Copyright by the Acoustical Society of America. Risi, J. D., Burdisso, R. A., \& Fuller, C. R. (1996). Analytical investigation of active control of radiated inlet fan noise. Journal of the Acoustical Society of America, 99(1), 408-416. doi: 10.1121/1.414552

\title{
Analytical investigation of active control of radiated inlet fan noise
}

\author{
John D. Risi, Ricardo A. Burdisso, and Chris R. Fuller \\ Vibration and Acoustics Laboratories, Department of Mechanical Engineering, Virginia Polytechnic \\ Institute and State University, Blacksburg, Virginia 24061-0238
}

(Received 17 October 1994; revised 4 May 1995; accepted 7 August 1995)

\begin{abstract}
An analytical model has been developed to study the potential of active noise control techniques for reducing radiated tonal inlet noise from turbofan engines. The analytical model consists of multiple control sources placed in the periphery of the engine inlet duct which inject antinoise into the duct to destructively interfere with the tonal sound field generated by the fan. The development of the analytical expressions of the radiated sound fields due to both the disturbance and the control sources is based on duct modal expansion. Attenuation of the radiated sound field is accomplished using a multiple-channel feedforward control approach. Control signals are calculated to minimize the pressure disturbance at desired error sensor locations and are then used to determine the controlled radiated sound field. The model is used to evaluate the performance of the control system for single and multiple circumferential arrays of control sources. The model can be extended for use in design optimization. Sample results are presented to illustrate the predicted sound attenuations. () 1996 Acoustical Society of America.
\end{abstract}

PACS numbers: $43.40 . \mathrm{Vn}$

\section{INTRODUCTION}

Noise is a significant negative factor associated with the modern commercial airline industry. When turbojets were first introduced, the primary source of noise was from the engine exhaust in the take-off configuration. The high power settings required for climb-out created a broadband exhaust noise that dominated in the vicinity of the take-off path of the aircraft. During approach, when the engine is at or near idle condition, the discrete high-frequency whine of the compressors becomes dominant. ${ }^{1}$ As engines evolved from turbojet to primarily turbofan cycles, fan noise has become an increasingly larger contributor of total engine noise. The impending use of ultrahigh bypass ratio engines will result in an even greater fan noise component at lower frequencies. Noise reduction techniques to date consist primarily of passive liners. However, the shorter inlet ducts inherent to the ultrahigh bypass engines and the lower blade passage frequencies expected for these engines will make passive attenuation of the inlet fan noise even more difficult. In order to meet forecast noise level requirements, the use of active noise control techniques may have the potential to overcome these problems.

The concept of active sound control, or antinoise as it is sometimes referred, is attributed to Lueg. ${ }^{2}$ Although Lueg's patent is almost 60 years old, only in the past 20 years has widespread use of active control begun to emerge. Very recently, an experimental effort has been undertaken by Thomas et al. ${ }^{3}$ to investigate the potential of active noise control approaches to reduce fan noise from a turbofan engine. In this work, the tonal noise radiated by the fan of a Pratt and Whitney JT15D turbofan engine was controlled using an adaptive feedforward control algorithm. The control source transducers were electromagnetic loudspeakers attached to the engine inlet duct through exponential horns while error microphones placed in the acoustic far field monitored the resultant sound field. The control algorithm adaptively determined the optimum control inputs necessary to minimize the sound-pressure levels at the error sensor locations. Reduction of up to $16 \mathrm{~dB}$ of the blade passage frequency tone in localized areas in front of the engine was demonstrated using a multiple-channel control system. Simultaneous control of multiple tones, i.e., fan blade passage frequency and the first harmonic, from the turbofan engine was also achieved.

More recently, Kraft and Kontos ${ }^{4}$ developed an analytical model to explore the theoretical implications of active control of turbofan engine noise. The secondary control source of their model is a single axial station comprised of electromagnetic sources located in the engine duct, flush with the outer wall of the inlet duct, coupled to the duct by a short horn. The authors assume a single mode to be present in the inlet duct. They study the required optimum number of active noise control sources needed to obtain global control of the sound field radiated from the engine inlet duct due to a single acoustic mode. The efficiency of conversion of electrical energy into acoustical energy for electromagnetic loudspeakers radiating into the duct was also addressed.

Berge et $a l .{ }^{5}$ used the boundary element method to evaluate the concept of active noise control for turbofan noise reduction. Their prime objective is not to reduce the acoustical field in all directions, but to reduce only certain regions of the far-field radiation which are of concern, such as the aircraft cabin or the flyover area of the aircraft. They accept the consequence that an increase in sound-pressure levels may occur and attempt to direct this higher sound energy to convenient domains.

In this paper, an analytical model is developed to predict the radiated acoustic field at a single frequency from both the engine inlet and an active noise control system. The engine inlet noise field is assumed to be composed of a known set of spinning modes. The control sources are modeled as point 


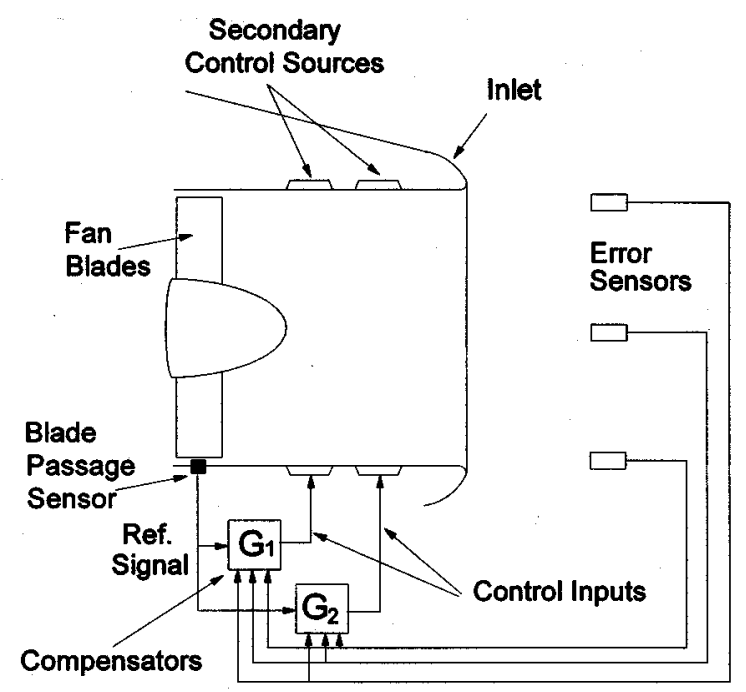

FIG. 1. Schematic of a feedforward active control system.

sources located on the periphery of the engine inlet duct, while the far-field error transducers are modeled as point microphones. The optimum control inputs to the secondary sources are obtained by minimizing the sum of the error signal mean-square values. These control inputs are then applied to the secondary sources to compute the controlled acoustic field. The main goal of this research is to analytically explore the potential of active noise control techniques in attenuating inlet noise. The analytical model development is predicated on incorporating into a simple model the fundamental aspects of the active control mechanism. At this stage it is not the purpose to produce a highly accurate model, i.e., a full aerodynamic model of the disturbance source mechanism and so forth; that in itself is a subject of research on its own.

\section{ANALYTICAL MODEL}

The analytical effort undertaken involves the mathematical modeling of the radiated sound field due to both the engine fan and the secondary control sources, and the simulation of the feedforward control system. Figure 1 shows a schematic of a feedforward control system. The rotating fan blades produce a disturbance sound field at the blade passage frequency (BPF) and its harmonics. This undesired noise field propagates down the inlet and radiates into the far field. A proximity sensor mounted on the engine at or near the fan station generates sinusoidal signals at the BPF and harmonics which are input into an array of compensators. The outputs from the compensators are the control signals. An array of secondary sources, driven by the control signals, generates the secondary sound field that destructively interferes with the disturbance noise field. The compensators that yield the optimum control inputs are determined so as to minimize the pressure at an array of error sensors which can also be installed in the inlet.

The engine inlet is modeled as a semi-infinite, open ended duct with rigid walls. The opening of the inlet is assumed to be embedded in an infinite baffle which simplifies the computation of the far-field radiation. In this initial study

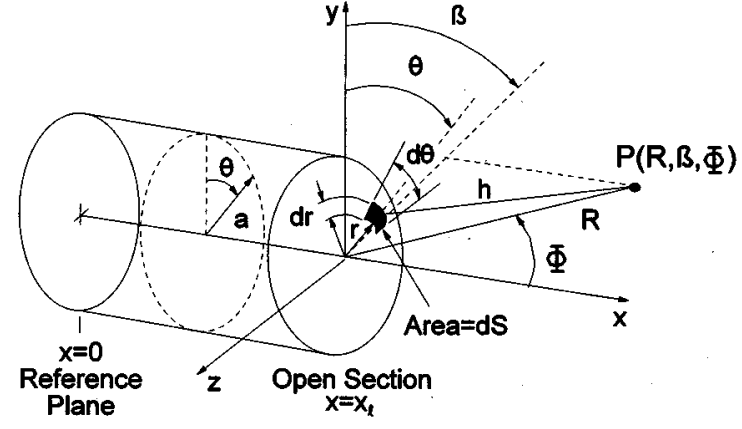

FIG. 2. Cylindrical coordinate system for inlet duct analysis and spherical coordinate system for far-field analysis.

the effect of mean flow is not considered. Due to the relatively high normalized wave numbers considered here, acoustic reflection from the duct opening is deemed negligible.

\section{A. Fan disturbance noise field}

The cylindrical coordinate system $(r, \theta, x)$ used in the analysis is shown in Fig. 2. The aerodynamic noise generation mechanism in turbomachinery is a subject of intensive research on its own and beyond the scope of this work. For the sake of simplicity, the fan is assumed to produce a known set of spinning acoustic modes at the fan BPF. An acoustic mode is defined by the circumferential order $m$, and the radial order $\mu$, written as $(m, \mu)$. Thus the acoustic mode of order $(0,0)$ describes the plane-wave mode.

The disturbance pressure field in a cylindrical duct of radius $a$ at the BPF can be expressed as the contribution of spinning modes or characteristic functions traveling in the positive $x$ direction. ${ }^{6,7}$ Without lost of generality, only modes rotating in the positive $\theta$ direction are considered. Thus

$$
\left(P_{i}\right)_{d}(r, \theta, x, t)=\sum_{m, \mu} A_{m \mu} J_{m}\left(k_{m \mu} r\right) e^{-i m \theta} e^{-i k_{x} x} e^{i \omega t},
$$

where $A_{m \mu}$ are the known complex amplitudes of the $(m, \mu)$ spinning mode pattern, $J_{m}$ is the $m$ th-order Bessel function, and $k_{x}$ is the axial wave number given by

$$
k_{x}=\sqrt{(\omega / c)^{2}-k_{m \mu}^{2}},
$$

where $\omega=\omega_{r} N_{b}$ is the fan BPF, $\omega_{r}$ is the rotating angular velocity of the fan, $N_{b}$ is the number of fan blades, and $c$ is the speed of sound in air. The modal wave number or eigenvalue $k_{m \mu}$ is determined by the system radial boundary conditions. Here, the rigid wall condition for the inlet duct implies that the radial component of the fluid particle velocity at $r=a$ must vanish. This leads $k_{m \mu}$ to be defined as

$$
k_{m \mu}=j_{m \mu}^{\prime} / a,
$$

where $j_{m \mu}^{\prime}$ are the inflection points of $J_{m}$ at $\left(d J_{m} / d x\right)=0$. $^{7}$ The propagation characteristic of the modes is determined by the axial wave number $k_{x}$. A mode will propagate for $k_{m \mu} \leqslant \omega / c$, and it will be attenuated when $k_{m \mu}>\omega / c$. The frequency at which this change occurs is called the cutoff 
frequency. Only the propagating modes are included in the expression of Eq. (1).

Setting the circumferential order $m$ to zero in Eq. (1) leads to the special case of the axisymetric modes, i.e., planewave and higher-order radial modes. In the sequel, the timedependent term $e^{i \omega t}$ will be omitted for simplicity.

\section{B. Point-source sound field}

The noise radiated by the fan is actively reduced by an array of $N_{s}$ control sources mounted in the duct wall. The sound field generated by the control sources will destructively interfere with the fan noise, thus reducing the inlet radiated far-field noise. The control sound source is modeled as an array of point sources distributed on the duct wall. Thus an expression for the acoustic field produced by a simple source located at the duct wall is required. This is approximated using Green's function on a hard walled infinite duct. Assuming the $n$th point source is located at $\left(a, \theta_{n}, x_{n}\right)$, the Green's function for a positive- $x$ traveling wave can be written as 8

$$
\begin{aligned}
\left(P_{i}\right)_{c}^{n}(r, \theta, x)= & \frac{-i}{2 \pi a^{2}} \sum_{m, \mu} \frac{\Psi_{m \mu}(r, \theta) \Psi_{m \mu}\left(a, \theta_{n}\right)}{\Lambda_{m \mu} k_{x}} \\
& \times e^{-i k_{x}\left|x_{l}-x_{n}\right|},
\end{aligned}
$$

where the duct acoustic mode shape is defined as

$$
\Psi_{m \mu}(r, \theta)=\cos \left(m\left(\theta-\theta_{n}\right)\right) J_{m}\left(k_{m \mu} r\right)
$$

and $\Lambda_{m \mu}$ is the mean-square amplitude of the cross-duct mode defined as

$$
\Lambda_{m \mu}=\frac{1}{\epsilon_{m}}\left(1-\frac{m^{2}}{\left(k_{m \mu} a\right)^{2}}\right) J_{m}^{2}\left(k_{m \mu} a\right),
$$

where $\epsilon_{m}=1$ for $m=0$ and $\epsilon_{m}=2$ for $m>0$.

\section{Radiated far-field pressure}

The sound field generated by both the fan and the control sources propagates along the duct to the open end at $x=x_{l}$ where it then radiates into the far field. The spherical coordinate system $(R, \beta, \Phi)$ used to compute the pressure radiated to the far field is shown in Fig. 2. The nonuniform pressure distribution at the duct opening can be determined using the expressions of Eqs. (1) and (4). To compute the radiated sound into the far field, the velocity fluctuation across the duct opening can be assumed to be volume velocity sources. ${ }^{9}$ Further assuming the duct to be embedded in a baffle, the pressure radiated to a point in the far field defined by $(R, \beta, \Phi)$ can be obtained by using the Rayleigh integral as $^{10}$

$$
P_{\mathrm{ff}}(R, \beta, \Phi)=\frac{i \omega \rho}{2 \pi} \int_{S} \nu_{x}(r, \theta) \frac{e^{-i k_{0} h}}{h} d S,
$$

where, referring to Fig. $2, R$ is the position vector of the observation point, $r$ is the position vector of the elemental surface $d S$ having normal velocity $\nu_{x}(r, \theta)$, and $h$ is the magnitude of the vector $R-r$. Also, $\rho$ is the density of air and $k_{0}=\omega / c$ is the acoustic wave number. Equation (7) assumes that the reflection from the duct opening is negligible.
This is a reasonable approximation except near the cutoff frequency of the modes. ${ }^{9}$ The fluid particle velocity normal to the duct opening needed in Eq. (7) is computed as

$$
\nu_{x}(r, \theta)=-\left.\frac{1}{i \omega \rho} \frac{\partial P}{\partial x}\right|_{x=x_{l}} .
$$

The far-field pressure due to the fan disturbance is then obtained by substituting Eq. (1) into (8) and this into the Rayleigh integral of Eq. (7). Referring to Fig. 2, an expression for the magnitude of the vector $R-r$ can be obtained using the far-field approximation $R \gg r$ as

$$
h \cong R-r \sin \Phi \cos (\theta-\beta) .
$$

Considering the approximation in Eq. (9), the pressure at $(R, \beta, \Phi)$ in the far field is given as

$$
\begin{aligned}
\left(P_{\mathrm{ff}}\right)_{d}= & \sum_{m, \mu} \frac{i A_{m \mu} k_{x}}{2 \pi R} e^{-i\left(k_{x} x_{l}+k_{0} R\right)} \\
& \times \int_{S} J_{m}\left(k_{m \mu} r\right) e^{-i m \theta} e^{i k_{0} r \sin \Phi \cos (\theta-\beta)} d S,
\end{aligned}
$$

where it has been further assumed that $h=R$ in the denominator of Eq. (7). The above integral has a closed-form solution, resulting in ${ }^{11}$

$$
\left(P_{\mathrm{ff}}\right)_{d}(R, \beta, \Phi)=\sum_{m, \mu} i^{m+1} \frac{k_{x} A_{m \mu}}{R} e^{-i\left(k_{x} x_{l}+k_{0} R\right)} e^{-i m \beta} I_{r},
$$

where $I_{r}$ is defined for $\left(k_{m \mu}\right)^{2} \neq\left(k_{0} \sin \Phi\right)^{2}$ as

$$
\begin{aligned}
I_{r}= & \frac{a}{\left(k_{m \mu}\right)^{2}-\left(k_{0} \sin \Phi\right)^{2}}\left\{k_{m \mu} J_{m}\left(k_{0} a \sin \Phi\right)\right. \\
& \times J_{m+1}\left(k_{m \mu} a\right) \\
& \left.-k_{0} \sin \Phi J_{m}\left(k_{m \mu} a\right) J_{m+1}\left(k_{0} a \sin \Phi\right)\right\}
\end{aligned}
$$

and for $\left(k_{m \mu}\right)^{2}=\left(k_{0} \sin \Phi\right)^{2}$ as

$$
\begin{aligned}
I_{r}= & \frac{a^{2}}{2}\left\{J_{m}^{2}\left(k_{m \mu} a\right)-\frac{2 m}{k_{m \mu} a} J_{m}\left(k_{m \mu} a\right) J_{m+1}\left(k_{m \mu} a\right)\right. \\
& \left.+J_{m+1}^{2}\left(k_{m \mu} a\right)\right\} .
\end{aligned}
$$

The far-field sound pressure due to a single source located within the duct is computed by replacing Eq. (4) into Eq. (8) and this into the Rayleigh integral of Eq. (7). Assuming again the far-field approximation of Eq. (9) yields

$$
\begin{aligned}
& \left(P_{\mathrm{ff}}\right)_{c}^{n}=\sum_{m \mu} \frac{\Psi_{m \mu}\left(a, \theta_{n}\right)}{4 \pi^{2} a^{2} R \Lambda_{m \mu}} e^{-i\left(k_{x}\left|x_{l}-x_{n}\right|+k_{0} R\right)}
\end{aligned}
$$

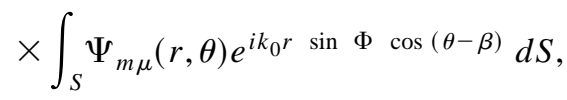

then, completing the integration and simplifying, the pressure at a point $(R, \beta, \Phi)$ in the far field due to a control source is ${ }^{11}$ 


$$
\begin{aligned}
\left(P_{\mathrm{ff}}\right)_{c}^{n}= & \sum_{m \mu} \frac{i^{m} J_{m}\left(k_{m \mu} a\right)}{2 \pi a^{2} R \Lambda_{m \mu}} \cos \left(m\left(\theta_{n}-\beta\right)\right) \\
& \times e^{-i\left(k_{x}\left|x_{l}-x_{n}\right|+k_{0} R\right)} I_{r},
\end{aligned}
$$

where $I_{r}$ is defined in Eqs. (12) and (13).

Equation (15) defines the far-field pressure due to a single control source. In many practical systems, an array of control sources will be grouped in a single channel driven by the same control signal. Thus the far-field pressure due to the point sources grouped into the $l$ th control channel can be written as

$$
\left(P_{\mathrm{ff}}\right)_{l}=\left\{R_{l}\right\}^{T}\left\{\left(P_{\mathrm{ff}}\right)_{c}\right\},
$$

where $\left\{\left(P_{\mathrm{ff}}\right)_{c}\right\}^{T}=\left\{\left(P_{\mathrm{ff}}\right)_{c}^{1}, \ldots,\left(P_{\mathrm{ff}}\right)_{c}^{N_{s}}\right\}$ is the vector of far-field pressure due to each point source computed from Eq. (15) and $\left\{R_{l}\right\}$ is the control source configuration vector for channel $l$. The elements in $\left\{R_{l}\right\}$ represent the magnitude and phase relative to each point source grouped into channel $l$ and driven by the $l$ th control amplitude $U_{l}$. In general, the coefficients of $\left\{R_{l}\right\}$ are complex numbers so that any relative magnitude and phase between the sources can be defined. However, in this work they will take the form of $1,-1$, and 0 representing in-phase, out-of-phase, or disconnected condition, respectively. The total far-field pressure due to the $N_{c}$ control channels can then be expressed as

$$
\left(P_{\mathrm{ff}}\right)_{c s}=\{U\}^{T}[R]\left\{\left(P_{\mathrm{ff}}\right)_{c}\right\},
$$

where $\{U\}^{T}=\left\{U_{1}, \ldots, U_{N_{c}}\right\}$ is the vector of $N_{c}$ control amplitudes and the matrix $[R]$ is the control configuration matrix for the $N_{c}$ control channels. The $l$ th row of this matrix is the vector $\left\{R_{l}\right\}$ previously described.

\section{Feedforward control theory}

The total radiated far-field pressure is computed by using the expressions in Eqs. (11) and (17) for both the fan and control source fields. The total sound pressure at a point in the far field is defined as

$$
\left(P_{\mathrm{ff}}\right)_{t}=\left(P_{\mathrm{ff}}\right)_{d}+\left(P_{\mathrm{ff}}\right)_{c s} .
$$

An array of $N_{E}$ microphone error sensors $\left(N_{E} \geqslant N_{C}\right)$ is assumed placed in the far field to monitor the resultant acoustic field. The use of far-field microphones as error sensors is certainly not feasible in practice. However, they are considered in this study for two reasons. First, since they directly observe the quantity to be minimized, i.e., far-field radiated pressure, the results can be used as a reference or upper limit performance bound. The performance of realistic sensing strategies can then be compared against this "optimal" farfield sensing strategy. Second, although no physical transducer can be placed in the far field, the far-field pressure can be estimated using transducers placed at other locations, i.e., on the fuselage or inside the inlet. The transfer functions between the transducers and the far-field pressure can be analytically or experimentally modeled. The outputs of the error transducers are then input into these transfer functions to predict the far-field pressure.
In feedforward control, the optimum complex control amplitudes $U_{l}$ are obtained by minimizing a cost function which is defined as the sum of the error sensor mean-square values. That is,

$$
C\left(U_{l}\right)=\sum_{e=1}^{N_{E}}\left(P_{\mathrm{ff}}\right)_{e}\left(P_{\mathrm{ff}}^{*}\right)_{e},
$$

where $\left(P_{\mathrm{ff}}\right)_{e}$ is the far-field pressure at the $e$ th error sensor location, evaluated using Eq. (18), and an asterisk $(*)$ denotes the complex conjugate. A thorough description of the control approach used in this work is beyond the scope of this publication. Hence only a brief overview is presented here. A complete review of the feedforward control approach is available in Risi. ${ }^{12}$

The cost function defined in Eq. (19) is a quadratic function of the control amplitude $U_{l}$. Differentiating the cost functions with respect to the real and imaginary parts of the control amplitude and setting them to zero, the optimum complex control amplitude vector is given by

$$
\{U\}=-\left([T]^{T}[T]^{-1}[T]^{T}\right)\left\{\left(P_{\mathrm{ff}}\right)_{d}^{e}\right\},
$$

where $\left(P_{\mathrm{ff}}\right)_{d}^{e}$ is the pressure at the $e$ th error sensor location due to fan noise; the $(e, l)$ elements of matrix $[T]$ represent the complex pressure at the $e$ th error sensor due to a unit amplitude of the $l$ th control channel. For the case of equal error sensors and control channels $\left(N_{E}=N_{C}\right)$, Eq. (20) reduces to

$$
\{U\}=-[T]^{-1}\left\{\left(P_{\mathrm{ff}}\right)_{d}^{e}\right\}
$$

and all the error signals are theoretically driven to zero.

\section{NUMERICAL EXAMPLES}

To illustrate the performance of the analytical model, an inlet diameter of $0.53 \mathrm{~m}$ and a duct length of $1 \mathrm{~m}$ is considered. The blade passage frequency is assumed to be $f_{\mathrm{BPF}}=2400 \mathrm{~Hz}$. These model parameters are based on the experimental work of Thomas et al. ${ }^{3}$ The speed of sound in air, $c$, is $343 \mathrm{~m} / \mathrm{s}$ and the density of air, $\rho$, is $1.21 \mathrm{~kg} / \mathrm{m}^{3}$. It is initially assumed that the fan produces only the first-order spinning circumferential modes, $m=1$. At the BPF selected only the first three radial modes $\mu=1,2,3$ propagate along the duct. The complex amplitude of these propagating modes needed in Eq. (1) can be determined from the aerodynamics of the fan system, which is beyond the scope of this work. For simplicity, it is assumed that all three modes have the same relative amplitude and phase, $A_{10}=A_{11}=A_{12}=1.0$.

\section{A. Single axial control source array}

The first control source configuration chosen is a sixchannel $\left(N_{C}=6\right)$ system comprised of 12 evenly distributed point sources $\left(N_{S}=12\right)$ located within the periphery of the inlet duct, with the first point source placed at $\theta=0^{\circ}$. The single axial plane of control sources is placed at $x=0.5 \mathrm{~m}$ upstream of the fan reference plane $x=0$. The first row of the control configuration matrix $[R]$ is $\left\{R_{1}\right\}=\{1,0,0,0,0,0,-1,0,0,0,0,0\}$, indicating that channel one, $U_{1}$, drives control sources 1 and 7 with a $180^{\circ}$ phase difference. Channel two is described by 


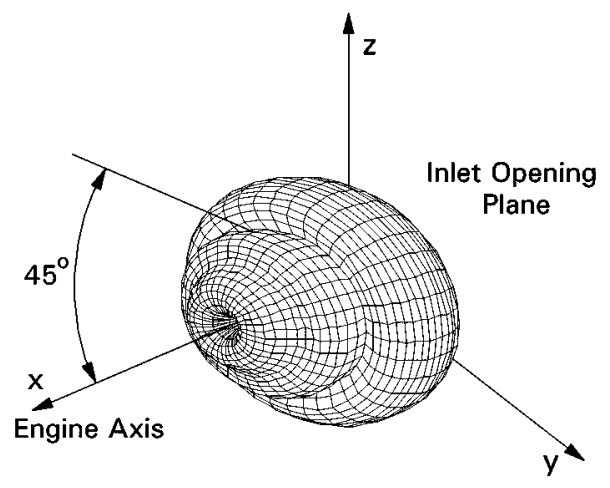

FIG. 3. Radiation directivity in $\mathrm{dB}$ of the uncontrolled fan noise for an engine modeled with an inlet diameter of $0.53 \mathrm{~m}$, a duct length of $1 \mathrm{~m}$, and a blade passage frequency of $2400 \mathrm{~Hz}$.

$\left\{R_{2}\right\}=\{0,1,0,0,0,0,0,-1,0,0,0,0\}$ indicating that control sources 2 and 8 are out of phase and driven by the second control signal $U_{2}$. The remainder of matrix $[R]$ follows the set pattern. This configuration is selected for best coupling with the $m=1$ duct modes. Each point source is assumed to excite all propagating modes at the fan BPF, $k_{m \mu} \leqslant \omega / c$.

Six error sensors $\left(N_{E}=6\right)$ are used to measure the farfield radiation. The six point error sensors are distributed around the engine centerline axis, with the first sensor at $\beta=0^{\circ}$ and each of the remaining sensors at $30^{\circ}$ intervals. Each error sensor is placed at an angular position of $\Phi=20^{\circ}$ off the engine axis, and at a radial distance $R=1.5 \mathrm{~m}$ from the open section of the duct. The axial location of the control source array and the angular position $\Phi$ of the error sensors were chosen to minimize the radiated power.

The resulting far-field radiation from the simulation of the fan disturbance alone, measured at a distance of $1.5 \mathrm{~m}$ from the duct opening, is shown in Fig. 3. This plot represents the three-dimensional (3-D) radiation directivity due to the fan in an uncontrolled state; i.e., the distance from the coordinate center to the surface represents the soundpressure level in decibels $(\mathrm{dB})$ in that direction at $R=1.5 \mathrm{~m}$. The $y-z$ plane of Fig. 3 represents the inlet opening plane of the engine. The existence of a notch at the center of the directivity pattern, i.e., along the $x$ axis, is clearly visible. A pressure decrease at approximately $45^{\circ}$ off the engine axis between two major lobe patterns as well as a lower pressure distribution of the fan noise at the extreme sidelines are clearly seen. The total radiated sound power level is 87.56 $\mathrm{dB}$.

Using the control source configuration and error sensor locations previously described, the complex control inputs are calculated using Eq. (21). These control inputs are plotted in the complex plane shown in Fig. 4. The control inputs display equal magnitude and an equal phase angle between consecutive inputs corresponding to the angular separation of the control channels. It is easy to show that this control system structure generates only first-order circumferential spinning mode patterns $(m=1$ and $\mu=1,2,3)$ spinning at frequency $\omega=2 \pi f_{\mathrm{BPF}}$. The implication of this result is that with a single circumferential array the higher-order radial modes are excited with a fixed magnitude and phase relative

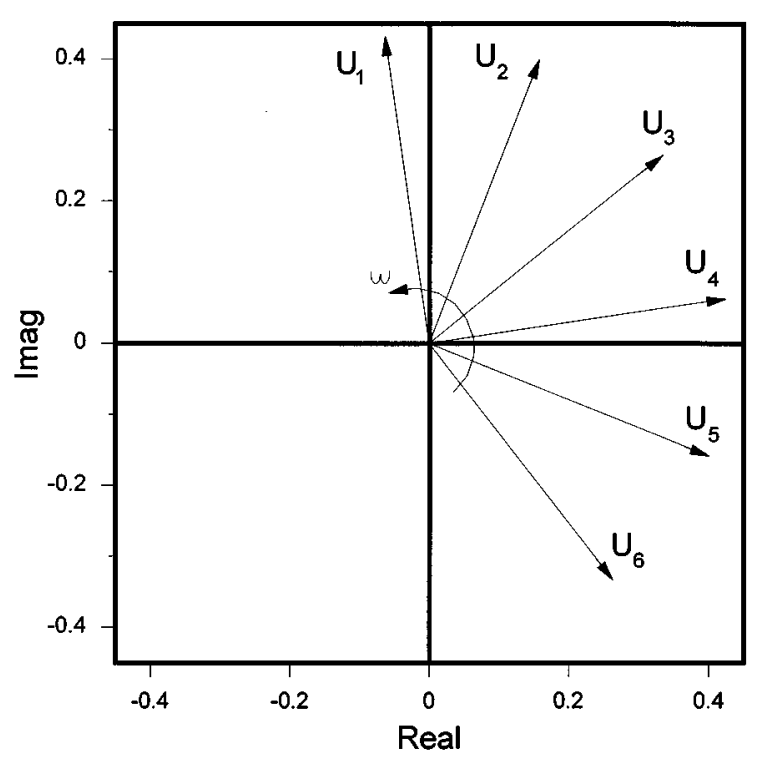

FIG. 4. Complex control inputs for single axial control source array configuration.

to each other. The magnitude and phase are directly determined by the control source location. In other words, the higher-order modes cannot be controlled independently with a single circumferential array.

The resulting 3-D radiation directivity for the controlled system is shown in Fig. 5. The symmetrical notch in the pressure pattern at $20^{\circ}$ off the engine axis is created by the controller driving the error sensor signals to zero. This 3-D view of the controlled engine noise reveals that the axisymetric pattern of the acoustic field has been preserved using the selected error sensor configuration. A vertical cross section taken from the radiation directivity plot is shown in Fig. 6. The solid line of this two-dimensional radiation plot represents the uncontrolled fan noise while the dashed line represents the controlled field. In Fig. 6, it can more clearly be seen that by placing the error sensors at $20^{\circ}$ off the engine axis the pressure in this region is reduced significantly. It is also evident that nearly global control of the fan noise is achieved. The total radiated sound power level with control is $81.18 \mathrm{~dB}$, giving a $6.38-\mathrm{dB}$ reduction in power.

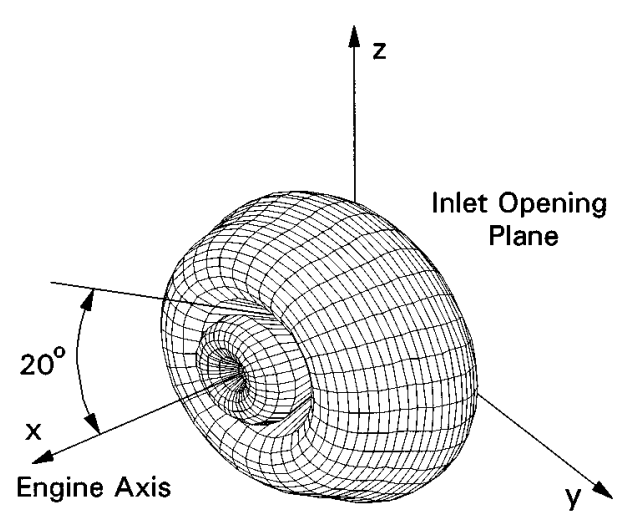

FIG. 5. Radiation directivity in $\mathrm{dB}$ of controlled fan noise using single axial control source array configuration. 


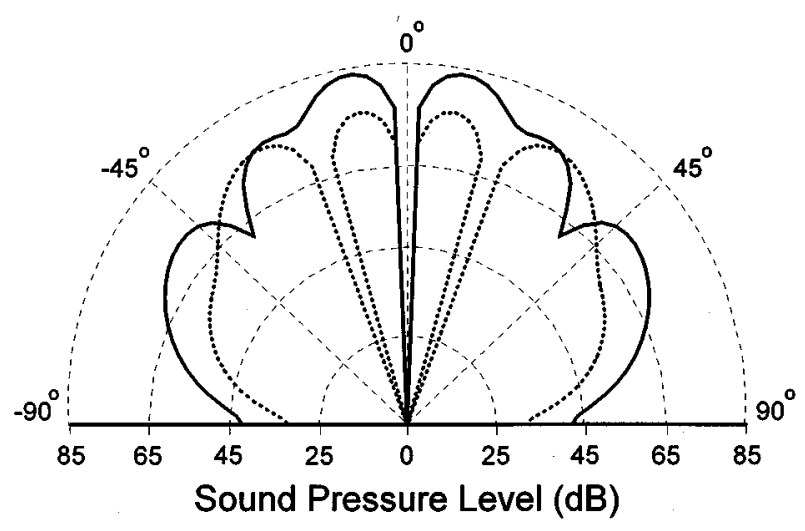

FIG. 6. Vertical cross section of radiation directivity of controlled fan noise for single axial control source array configuration.

\section{B. Multiple axial control source configuration}

As mentioned before, a single circumferential array of control sources does not allow control of each higher-order radial mode independently. Thus the potential of improving the reduction of fan noise using multiple circumferential arrays, and thus increasing the flexibility in the independent excitation of the radial modes, was next investigated. Here, two axial stations, each consisting of 12 point sources, are employed. The original control source station, comprised of channels $1-6$, is moved to an axial location $0.6 \mathrm{~m}$ upstream of the fan. The second control source station, comprised of channels $7-12$, is placed $0.85 \mathrm{~m}$ upstream of the fan and is configured similarly to the original band. Six additional error sensors are also required. These point sensors are placed in the same pattern around the circumference of the engine as the original set. The first set of error sensors are moved to an angular position of $10^{\circ}$ off the centerline axis and the second set of point sources are placed $40^{\circ}$ off the centerline axis. Again, this actuator and sensor configuration was selected to minimize the radiated power. The complex control inputs of this 12-channel system are presented in Fig. 7. The control inputs reveal that a spinning mode pattern has been achieved by each axial station of control sources, with the magnitudes of each set differing due to the location of each band within the duct. Unlike the case of a single control station, multiple axial control stations allow for the independent control of the higher-order radial modes. A vertical cross section taken of the radiation directivity, seen in Fig. 8, reveals that global control has been achieved. Notches at the error sensor locations are clearly seen. The sound power level for this multiple circumferential array configuration is $59.57 \mathrm{~dB}$, which reveals that the two circumferential array of control sources performs significantly better, with a resulting sound power level of nearly $22 \mathrm{~dB}$ less than that of the single circumferential array configuration. The use of three axial array stations reveals total cancellation of the radiated sound. This is because fully independent control of the magnitude and phase of the higher-order radial modes is accomplished.

To explore the sensitivity of the system configuration, the far-field error sensor configuration for the case of a single axial control source array is modified. The error sensor originally placed at $\beta=0^{\circ}$ and $\Phi=20^{\circ}$ is moved to a new arbi-

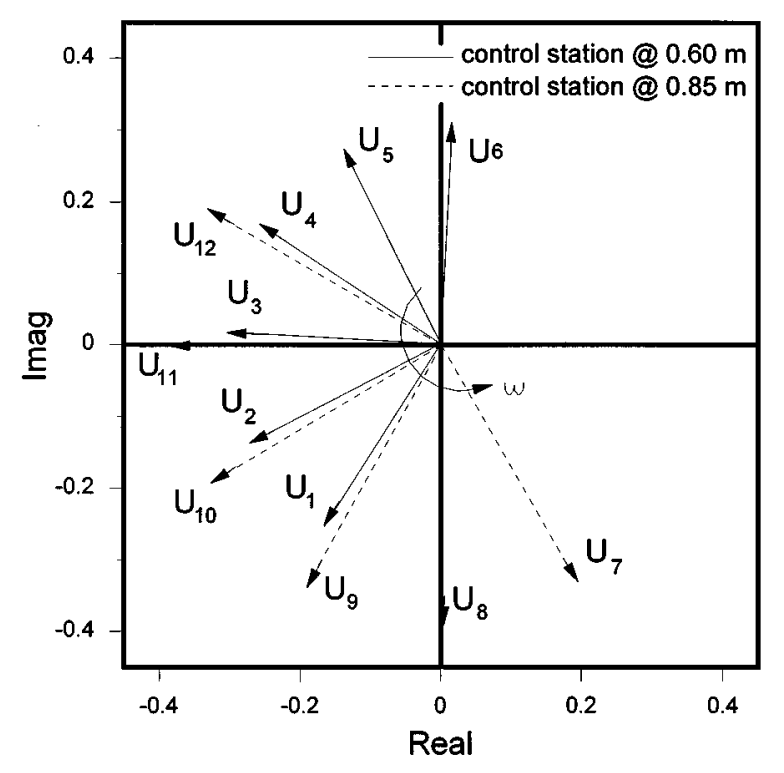

FIG. 7. Complex control inputs for multiple axial control source array configuration.

trarily selected angular position of $\Phi=30^{\circ}$. The new position of the error sensor is $0.26 \mathrm{~m}(1.83 \times$ the acoustic wavelength, $c / f)$ from its previous position. All other system parameters are maintained. The complex control inputs are presented in Fig. 9 and show that the symmetry in the control inputs was destroyed. This implies that the total control sound field is now made up of many duct modes which do not create spinning patterns. Moreover, Fig. 9 shows large discrepancies in the control input amplitudes. This implies that some secondary control sources will be operated below their full capacities while others will be driven to their limit. This clearly would be an inefficient use of the secondary control sources.

The resulting 3-D controlled far-field radiation directivity for the modified system configuration is shown in Fig. 10. The feature most obvious in this figure is the nonsymmetrical result of this modified control scheme. The pressure distribution reveals that only localized pressure reduction is realized, occurring on or very near the error sensors, as well as areas with significant spillover effects. The total radiated power of this modified configuration is $82.24 \mathrm{~dB}$. Figure 11 displays

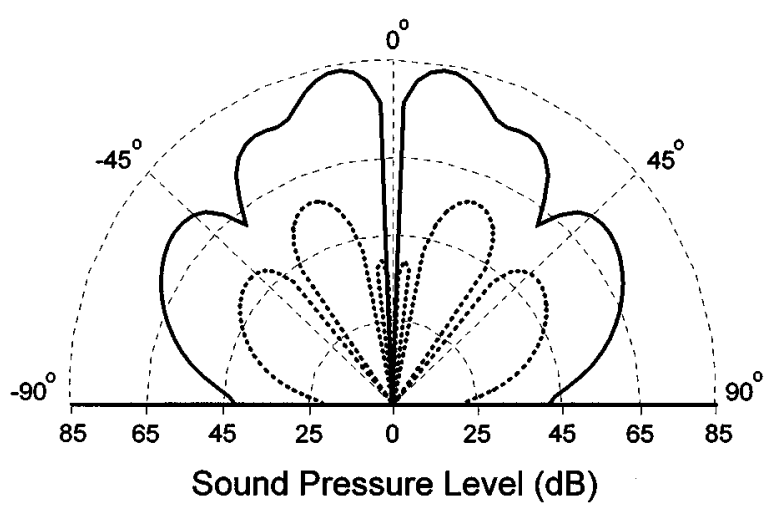

FIG. 8. Vertical cross section of radiation directivity of controlled fan noise using multiple control source array configuration. 


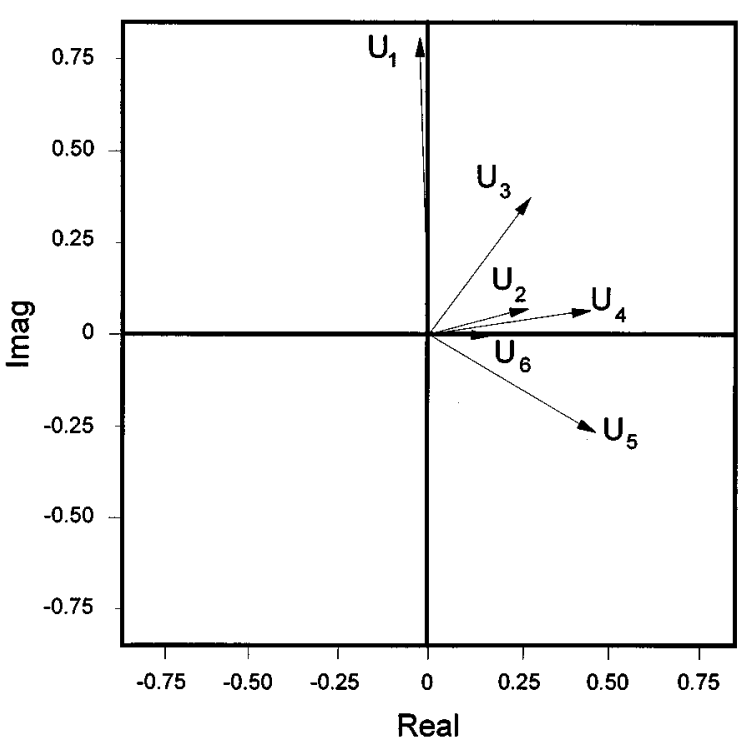

FIG. 9. Complex control inputs for modified single axial control source configuration.

the vertical cross section of the radiation directivity. The pressure notch at the error sensor location is visible, along with a small reduction of the fan noise in the center regions. An increase in the radiated pressure, or spillover, is seen over the sideline area. This spillover is mainly due to the radiation from the higher-order modes excited by the control sources in particular if they are near the cutoff frequency.

It is important to remark that similar behavior in the controlled acoustic field is observed due to variations in other system parameters such as relative source strength, phase, location, and so forth. A complete sensitivity analysis will be the subject of future publications. However, the example presented here shows that these factors will have to be taken into account in the practical implementation of active control of inlet noise.

\section{CONCLUSIONS}

An analytical model has been developed to predict the resulting far-field radiation from a turbofan engine inlet with an active noise control system. Feedforward is the control approach used in this work. The main goal is to use a simple model to uncover the fundamental control mechanism and

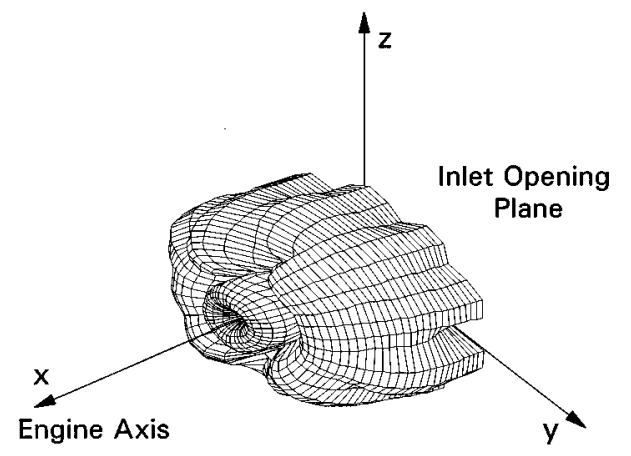

FIG. 10. Radiation directivity in $\mathrm{dB}$ of controlled fan noise using modified single axial array configuration.

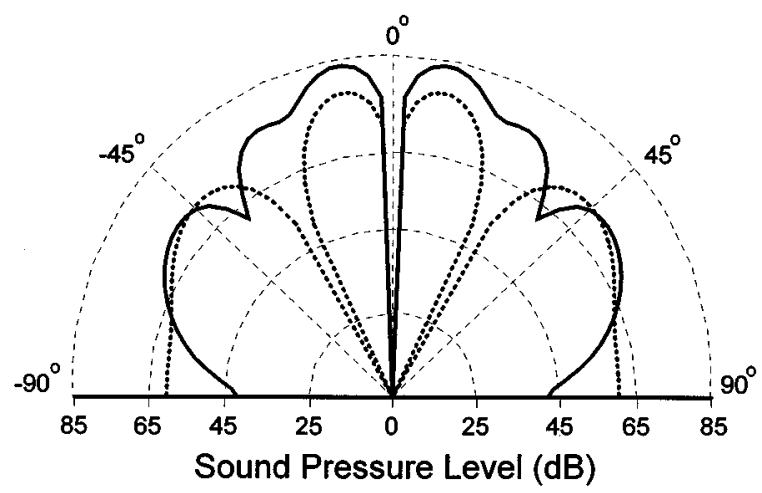

FIG. 11. Vertical cross section of radiation directivity of controlled fan noise using modified single axial control source configuration.

the behavior of the controlled system. Numerical results were obtained for controlling the first-order circumferential spinning modes. The first configuration consisted of a single circumferential array of 12 secondary sources evenly distributed around the inlet. The error sensors considered in this study are far-field error microphones. Proper placement of the error sensors and control sources resulted in nearly global attenuation of the radiated inlet noise at the blade passage frequency. The reduction in the radiated power is $6.38 \mathrm{~dB}$. However, the performance of the control system is sensitive to changes in control parameters. It is shown that changing the location of a single error sensor resulted in spillover to the sidelines with little reduction in the total radiated power. Furthermore, the axisymmetry of the problem was destroyed corresponding to an inefficient use of the secondary control sources. Using multiple circumferential arrays of control sources, an improvement in the global reduction was demonstrated with a reduction of $27.99 \mathrm{~dB}$ in the radiated power. This improvement in global reduction is due to the fact that multiple axial control source stations are required to independently control the higher radial order modes.

\section{ACKNOWLEDGMENT}

The authors gratefully acknowledge the support of this work by the Aeroacoustic Branch of NASA Langley Research Center under Grant No. 230-11-110F-107-3122871.

\section{APPENDIX A: POSITION VECTOR}

From Fig. 2, an expression for the magnitude of the vector $R-r$ can be defined as

$$
\mathbf{h}=\mathbf{R}-\mathbf{r} \text {. }
$$

Defining each vector $R$ and $r$ :

$$
\begin{aligned}
\mathbf{R}= & R \cos (\Phi) \hat{i}+R \sin \Phi \sin \beta \hat{j} \\
& +R \sin \Phi \cos \beta \hat{k}, \\
\mathbf{r}=r & \sin \theta \hat{j}+r \cos \theta \hat{k} .
\end{aligned}
$$

Substituting Eqs. (A2) and (A3) into Eq. (A1) and combining like terms yields 


$$
\begin{aligned}
\mathbf{h}= & R \cos \Phi \hat{i}+(R \sin \Phi \sin \beta-r \sin \theta) \hat{j} \\
& +(R \sin \Phi \cos \beta-r \cos \theta) \hat{k} .
\end{aligned}
$$

Squaring both sides of Eq. (A4) and simplifying, the vector $h$ can be written as

$$
h^{2}=R^{2}\left(1+\frac{r^{2}}{R^{2}}-\frac{2 r}{R} \sin \Phi \cos (\theta-\beta)\right) .
$$

Assuming the far-field approximation $R \gg r$, the term $r^{2} / R^{2}$ goes to zero. Then, Eq. (A5) can be written as

$$
h=R\left(1-\frac{2 r}{R} \sin \Phi \cos (\theta-\beta)\right)^{1 / 2} .
$$

Using the expansion series

$$
(1-x)^{1 / 2}=1-\frac{1}{2} x-\frac{1}{8} x^{2}-\cdots
$$

and eliminating higher-order terms, Eq. (A6) becomes

$$
h=R-r \sin \Phi \cos (\theta-\beta) .
$$

\section{APPENDIX B: RADIATED FAR-FIELD PRESSURE}

\section{Fan noise}

The pressure radiated to the far field due to the fan noise [Eq. (10)] can be rewritten by referring to Fig. 2 as

$$
\begin{aligned}
\left(P_{\mathrm{ff}}\right)_{d}= & \sum_{m, \mu} \frac{i A_{m \mu} k_{x}}{2 \pi R} e^{-i\left(k_{x} x_{l}+k_{0} R\right)} \int_{0}^{2 \pi} \int_{0}^{a} J_{m}\left(k_{m \mu} r\right) \\
& \times e^{-i m \theta} e^{i k_{0} \sin \Phi \cos (\theta-\beta)} r d r d \theta .
\end{aligned}
$$

Breaking and expanding the double integral of Eq. (B1), the integral in terms of $\theta$ is

$$
I_{\theta}=\int_{0}^{2 \pi}[\cos (m \theta)-i \sin (m \theta)] e^{i k_{0} r \sin \Phi \cos (\theta-\beta)} d \theta .
$$

The exponential term of Eq. (B2) can be expanded in terms of Bessel functions as ${ }^{11}$

$$
\begin{aligned}
e^{i k_{0} r \sin \Phi \cos (\theta-\beta)}= & J_{0}\left(k_{0} r \sin \Phi\right)+2 i^{m} J_{m}\left(k_{0} r \sin \Phi\right) \\
& \times \cos (m(\theta-\beta)) .
\end{aligned}
$$

Substituting the expansion of Eq. (B3) into Eq. (B2) and eliminating the term that vanishes at the limits of integration gives

$$
\begin{aligned}
I_{\theta}= & 2 i^{m} J_{m}\left(k_{0} r \sin \Phi\right)\left\{\int_{0}^{2 \pi} \cos (m(\theta-\beta))\right. \\
& \times \cos (m \theta) d \theta-i \int_{0}^{2 \pi} \cos (m(\theta-\beta)) \\
& \times \sin (m \theta) d \theta\} .
\end{aligned}
$$

Applying trigonometric identities to Eq. (B4) and completing the integration, the integral in terms of $\theta$ of Eq. (B2) becomes

$$
I_{\theta}=2 \pi i^{m} J_{m}\left(k_{0} r \sin \Phi\right)[\cos (m \beta)-i \sin (m \beta)]
$$

or, simplified to exponential form,

$$
I_{\theta}=2 \pi i^{m} J_{m}\left(k_{0} r \sin \Phi\right) e^{-i m \beta} .
$$

Substituting Eq. (B6) into Eq. (B1) yields

$$
\begin{aligned}
\left(P_{\mathrm{ff}}\right)_{d}= & \sum_{m, \mu} \frac{i^{m+1} k_{x}}{R} A_{m \mu} e^{-i\left(k_{x} x_{l}+k_{0} R+m \beta\right)} \\
& \times \int_{0}^{a} J_{m}\left(k_{m \mu} r\right) J_{m}\left(k_{0} r \sin \Phi\right) r d r .
\end{aligned}
$$

The integral of Eq. (B7) is now in terms of $r$ only. Since this integral is the product of two Bessel functions, the integral in terms of $r$ of Eq. (B7) can be written as ${ }^{11}$

$$
\begin{aligned}
I_{r}= & \frac{a}{\left(k_{m \mu}\right)^{2}-\left(k_{0} \sin \Phi\right)^{2}}\left\{k_{m \mu} J_{m}\left(k_{0} a \sin \Phi\right)\right. \\
& \times J_{m+1}\left(k_{m \mu} a\right) \\
& \left.-k_{0} \sin \Phi, J_{m}\left(k_{m \mu} a\right) J_{m+1}\left(k_{0} a \sin \Phi\right)\right\} .
\end{aligned}
$$

Equation (B8) is valid only for $\left(k_{m \mu}\right)^{2} \neq\left(k_{0} \sin \Phi\right)^{2}$. When this does not hold true, the integral of Eq. (B7) becomes

$$
\begin{aligned}
I_{r}= & \frac{a^{2}}{2}\left\{J_{m}^{2}\left(k_{m \mu} a\right)-\frac{2 m}{k_{m \mu} a} J_{m}\left(k_{m \mu} a\right) J_{m+1}\left(k_{m \mu} a\right)\right. \\
& \left.+J_{m+1}^{2}\left(k_{m \mu} a\right)\right\} .
\end{aligned}
$$

\section{Control source noise}

The pressure radiated to the far field due to the control sources [Eq. (14)] can be written as

$$
\begin{aligned}
\left(P_{\mathrm{ff}}\right)_{c}^{n}= & \sum_{m \mu} \frac{J_{m}\left(k_{m \mu} a\right)}{4 \pi^{2} a^{2} R \Lambda_{m \mu}} e^{-i\left(k_{x}\left|x_{l}-x_{n}\right|+k_{0} R\right)} \\
& \times \int_{0}^{2 \pi} \int_{0}^{a} \Psi_{m \mu}(r, \theta) e^{i k_{0} r \sin \Phi \cos (\theta-\beta)} r d r d \theta .
\end{aligned}
$$

Recalling the definition of the acoustic mode shape of Eq. (5), the double integral of Eq. (B10) can be written as

$$
\begin{aligned}
I_{r, \theta}= & \int_{0}^{2 \pi} \int_{0}^{a} \cos \left(m\left(\theta-\theta_{n}\right)\right) \\
& \times J_{m}\left(k_{m \mu} r\right) e^{i k_{0} r \sin \Phi \cos (\theta-\beta)} r d r d \theta .
\end{aligned}
$$

Separating the integrals of Eq. (B11), the integral in terms of $\theta$ becomes

$$
I_{\theta}=\int_{0}^{2 \pi} \cos \left(m\left(\theta-\theta_{n}\right)\right) e^{i k_{0} r \sin \Phi \cos (\theta-\beta)} d \theta .
$$

Applying trigonometric identities to the cosine term of Eq. (B12), the integral can be written as

$$
\begin{aligned}
I_{\theta}= & \int_{0}^{2 \pi}\left[\cos \left(m \theta_{n}\right) \cos (m \theta)\right. \\
& \left.+\sin \left(m \theta_{n}\right) \sin (m \theta)\right] e^{i k_{0} r \sin \Phi \cos (\theta-\beta)} d \theta .
\end{aligned}
$$


The terms within the integral of Eq. (B13) are analogous to those in Eq. (B2). Therefore, using similar operations applied to the fan noise, the integral in terms of $\theta$ for the control source noise becomes

$$
I_{\theta}=2 \pi i^{m} J_{m}\left(k_{0} r \sin \Phi\right) \cos \left(m\left(\theta_{n}-\beta\right)\right) .
$$

Substituting Eq. (B14) into Eq. (B11) and this into Eq. (B10), the pressure in the far field due to the control sources becomes

$$
\begin{aligned}
\left(P_{\mathrm{ff}}\right)_{c}^{n}= & \sum_{m \mu} \frac{i^{m}}{2 \pi a^{2} R \Lambda_{m \mu}} J_{m}\left(k_{m \mu} a\right) e^{-i\left(k_{x}\left|x_{l}-x_{n}\right|+k_{0} R\right)} \\
& \times \cos \left(m\left(\theta_{n}-\beta\right)\right) \int_{0}^{a} J_{m}\left(k_{m \mu} r\right) J_{m} \\
& \times\left(k_{0} r \sin \Phi\right) r d r .
\end{aligned}
$$

The integral in Eq. (B15) is now in terms of $r$ only and is exactly the form of the integral of Eq. (B7). Therefore, the solution of the integral in terms of $r$ of Eq. (B15) is Eq. (B8) for $\left(k_{m \mu}\right)^{2} \neq\left(k_{0} \sin \Phi\right)^{2}$ and Eq. (B9) for
$\left(k_{m \mu}\right)^{2}=\left(k_{0} \sin \Phi\right)^{2}$.
${ }^{1}$ J. E. Peterson, Airports for Jets (Blakely-Oswald, Chicago, 1959).

${ }^{2}$ P. Lueg, "Process of silencing sound oscillations," US Patent No. 2,043,416 (1934).

${ }^{3}$ R. H. Thomas, R. A. Burdisso, C. R. Fuller, and W. F. O'Brien, “Active Control of Fan Noise form a Turbofan Engine," AIAA Paper No. 93-0597, January 1993.

${ }^{4}$ R. E. Kroft and K. B. Kontos, "Theoretical Implications of Active Noise Control for Turbofan Engines," AIAA Paper No. 93-4355, October 1993.

${ }^{5}$ D. Berge, E. Bouty, and J. M. Cailleau, “Active Noise Control of a Jet Engine at Low Frequency Radiation: B.E.M. for Predicting Far-Field Noise Radiation," AIAA Paper No. 93-4354, October 1993.

${ }^{6}$ J. M. Tyler and T. G. Sofrin, "Axial Flow Compressor Noise Studies," SAE Trans. No. 70, 1962, pp. 309-332.

${ }^{7}$ L. E. Kinsler, A. R. Frey, A. B. Coppens, and J. V. Sanders, Fundamentals of Acoustics (Wiley, New York, 1982).

${ }^{8}$ P. M. Morse and K. U. Ingard, Theoretical Acoustics (Cambridge U.P., London, 1986).

${ }^{9}$ C. L. Morfey, "A Note on the Radiation Efficiency of Acoustic Duct Modes," J. Sound Vib. 9, 367-372 (1969).

${ }^{10}$ F. Fahy, Sound and Structural Vibration (Academic, New York, 1989).

${ }^{11}$ N. W. McLachlan, Bessel Functions for Engineers (Oxford, New York, 1934).

${ }^{12}$ J. D. Risi, “Analytical Investigation of Active Control of Radiated Inlet Noise from Turbofan Engines," Masters thesis, Virginia Polytechnic Institute and State University, Blacksburg, Virginia, May 1995. 